\title{
A Comparative Study of the Clinical Results of Two Treatment Methods for Spiral Fracture of the Lower Third of the Tibia Shaft Combined with Posterior Malleolar Fracture
}

\author{
Bang-jun Cheng ${ }^{a}$, Yi Luo ${ }^{\text {a* }}$, Jun-wu Huang ${ }^{\mathrm{a}}$, Xiao-Bing Feng ${ }^{\mathrm{a}}$ \\ ${ }^{a}$ Department of Orthopedics, Jin Shan Branch Hospital of Sixth People's Hospital of Shanghai, Shanghai 201599, China.
}

\begin{abstract}
Objective: To compare and analyze the clinical efficacy of spiral fracture of the lower third of the tibia shaft combined with a posterior malleolar fracture treated with a plate or hollow screws.

Methods: Surgical treatment was carried out in 61 patients with a spiral fracture of the lower third of the tibia shaft combined with a posterior malleolar fracture. After the tibia fractures were fixed with a plate, the posterior malleolus fractures were repaired using either three hollow screws (hollow screw group, 34 cases) or a plate (plate group, 27 cases). The ankle and foot scoring system recommended by Baird-Jackson was used to evaluate the ankle postoperatively.

Results: All 61 patients were followed-up; the follow-up period ranged from 6-36 months, with an average of 20.8 months. All fractures healed well, and no delayed unions or nonunions occurred. The function of the ankle joint according to the Baird-Jackson score system evaluation was as follows: in the hollow screw group, 18 were excellent, 10 were good, 5 were fair, and 1 was poor, with at least $82.4 \%$ experiencing good or excellent outcomes. In the plate group, 8 were excellent, 9 were good, 3 were fair, and 1 was poor, with $85.2 \%$ reporting excellent or good results. The difference in these two groups of Baird-Jackson scores with an excellent rate was not statistically significant $(\mathrm{P}=0.21>0.05)$.

Conclusion: Spiral fractures of the distal third of the tibia are often accompanied by posterior malleolar fractures. Clinical treatment of the posterior malleolar fracture had no obvious impact on the clinical curative effect of hollow screw and L-type plates. Slight trauma was observed with hollow screw internal fixation, while internal plate fixation was more reliable.

Keywords: Spiral tibia fracture; Posterior malleolar fracture; Hollow screw; Plate; Internal Fixation
\end{abstract}

\section{INTRODUCTION}

Spiral fracture of the distal third of the tibia along with posterior malleolus fracture is a unique type of complex fracture with a high incidence that is seen regularly in clinical practice. Because a posterior malleolus fracture is easily concealed, the displacement is often not obvious. Conventional plain film radiography does not make it easy to identify, so the clinical misdiagnosis rate is high. At present, there is no standard treatment for this type of posterior ankle fracture. From January 2010 to June 2016, 61 cases of spiral fracture of the distal third of the tibia along with ankle fracture of the posterior malleolus were repaired using medial ana-

*Corresponding author: Yi Luo

Mailing address: Department of Orthopedics, Jin Shan Branch Hospital of Sixth People's Hospital of Shanghai, Shanghai 201599, China.

E-mail: luoyii2005@126.com

Received: 20 July 2018 Accepted: 25 September 2018 tomic plate fixation at our hospital. Three cannulated screws were used in the treatment of 27 cases, and the remaining 34 cases were treated with an L-type steel plate; a comparison was made between the two treatment methods to determine which was most effective.

\section{CLINICAL DATA}

\section{General information}

Out of the 61 cases with this type of injury, 34 were treated with three cannulated screws. There were 23 males and 11 females who ranged in age from 27-71 years old (average: 38.4 years). Three of these fractures were open; 18 cases also presented with a proximal fibula fracture, while a distal fibula fracture was diagnosed in 7 cases. The causes of these injuries were traffic accidents in 22 patients, sprains in 7, and other causes in the remaining 5. In the treatment group, 27 cases were repaired using a steel plate. Nineteen of these were male, and eight were female. This group ranged in age from 26-68 years, with an average age 
of 39.2 years. Only one patient in this group had an open fracture. However, 13 presented with a proximal fibular fracture, and 8 also suffered a distal fibular fracture. The causes of these injuries were traffic accidents in 17 patients, sprains in 6 , and other causes in the remaining 4 . The size of the fractured fragment in these two groups ranged from $25-50 \%$ of the total size of the posterior malleolus.

Operative methods

All patients received epidural anesthesia and a lower limb tourniquet. All cases were treated with steel plate fixation of the tibial fractures. In the treatment group, a hollow screw with a length of about $10 \mathrm{~cm}$ was wound along the midline between the lateral malleolus and the Achilles tendon's trailing edge. When distal fibula fractures were present, fibula fixation was carried out using the same surgical wound. During subcutaneous tissue layer separation, close attention was paid to the protection of the Achilles tendon and the lateral sural nerve. The deep fascia was incised to expose the peroneal tendon. After lateral retraction, the tendon was exposed below the hallux longus and then retracted medially to reveal the distal tibia and posterior malleolus fracture block Volkmann. If a conversion to an open reduction and temporary fixation were required, an assessment of the reduction was carried out using needle-guided, intraoperative $\mathrm{C}$ arm fluoroscopy to determine the block level of the articular surface. When the surgeon was satisfied with the hollow drill-guided pinhole, the guide wire insertion proceeded for the three 4.0-mm hollow screws. A small fracture block often makes it difficult to insert three cannulated screws to eliminate the test. In the cannulated screw fixation group, the fracture block was fixed under direct visualization and was temporarily fixed using Kirschner wire. The level of reduction of the articular surface was evaluated intraoperatively using $\mathrm{C}$ arm fluoroscopy.

\section{Postoperative treatment}

Two groups of patients were treated without external fixation (such as plaster) and with antibiotics to prevent infection within 24 hours postoperatively. On the third day following surgery, patients were allowed to participate in passive and active functional ankle exercises. They were not allowed to bear any weight at all on the ankle until 4 weeks after the operation. At 8 weeks following surgery, participants could take part in limited weight bearing with protection. They were only cleared for full weight bearing on the ankle 12 weeks postoperatively if an imaging examination showed that the fracture had healed sufficiently.

\section{Efficacy evaluation criteria}

The function of the ankle joint was assessed according to the Baird-Jackson scoring system ${ }^{[1]}$. The results from the $\mathrm{x}$-ray (25 points), stability (15 points), walking ability (15 points), pain (15 points), activity (10 points), ability to work (10 points), and running capacity (10 points) scores were used to evaluate ankle function to generate a total out of a highest possible score of 100 points. A total score in the $96-100$ range was classified as excellent, which meant that the ankle joint had recovered completely without pain and patients had no activity restrictions. An overall score from 91-95 was classified as good, where the ankle's range of motion for ankle flexion and extension was normal or limited by no more than 15 degrees. These patients reported being tired or having occasional pain with activity but had no activity or work restrictions. A fair total score ranged from 91-80; at this score, patients experienced ankle joint movement limitations of less than 30 degrees and occasional pain. They were allowed to engage in light physical labor. All scores $<80$ points were classified as poor because patients often experienced pain and had limited joint motion greater than 30 degrees.

\section{Statistical methods}

Data were statistically analyzed using the Statistical Package for the Social Sciences (SPSS), version 12.0, statistical software for Windows. Measurement data were reported as the mean and standard deviation $(x \pm S)$. If the data were normally distributed, group-designed $t$ tests were used. Count data were analyzed using $\chi^{2}$ test, and a P-value $<0.05$ indicated statistical significance.

\section{RESULTS}

All 61 cases were followed up for 6-36 months (mean: 20.8 months). The infection rate of the cannulated screw group $(0.03 \%)$ was slightly lower than that of the steel plate group $(0.04 \%)$, but there was no significant difference in the infection rate between the two groups $\left(\chi^{2}=1.31, P=0.13>0.05\right)$. Both groups of fractures healed well with no cases experiencing a delayed union or nonunion. The average healing time was 7.3 weeks in the hollow nail group and 7.5 weeks in the steel plate group. There was no significant difference in the healing time between the two groups $(\mathrm{t}=1.25$, $\mathrm{P}=0.09>0.05$ ). No significant reduction of ankle fracture was found during follow-up. Ankle joint function was assessed at the time of the last follow-up visit using the Baird-Jackson scoring system. The cannulated screw group had 18 cases with excellent function, 10 with good function, 5 with fair function, and 1 with 
poor function. The rate of patients who reported good function or better was $82.4 \%$. Ankle function in the steel plate group was excellent in 14 cases, good in 9, fair in 3, and poor in 1 case, with $85.2 \%$ reporting good or excellent function. There was no significant difference between these two groups in terms of their Baird-Jackson score $\left(\chi^{2}=1.53, \mathrm{P}=0.21>0.05\right)$. In the plate group, one case of postoperative vertical toe deformity appeared with flexor pollicis longus muscle spasms. The condition had improved after plate removal and was no longer obvious.

\section{ANALYSIS AND DISCUSSION}

An overview of spiral fracture of the lower third of the tibia and posterior malleolus fracturen

In routine clinical practice, spiral fractures of the lower third of the tibia and ankle fractures are very common, but spiral fracture of the lower third of the tibia combined with an ankle fracture are not easy to identify using conventional plain x-ray film. Undisplaced fractures, which are often clinically misdiagnosed, are reported in spiral fractures of the distal third of the tibia with posterior malleolar fracture Department of Radiology, diagnostic rate is $67.9 \%$ leakage, physicians clinical Department of orthopedics the misdiagnosis rate of $53.6 \%{ }^{[2]}$. Therefore, all patients with a spiral fracture of the distal third of the tibia should undergo computed tomography (CT) or magnetic resonance imaging (MRI) of the ankle joint, as the incidence of ankle fracture in these patients is about $81.7 \%{ }^{[3]}$. The report of the study on the fracture of the tibia and the fracture of the posterior malleolus of the tibia in the spiral fracture of the tibia $9.7 \%{ }^{[2]}$. Stuermer et al ${ }^{[4]}$ reported that
$3.7 \%$ of tibial fractures were also combined with ankle fractures. Kukkonen et al. ${ }^{[5]}$ found that approximately $83.3 \%$ of tibial shaft fractures and posterior malleolus fractures were associated with fractures of the tibia. In the present study, the incidence of spiral fracture of the distal third of the tibia and concurrent ankle fracture was about $76.1 \%$ after CT or MRI examination.

At present, there is no definitive treatment for this type of fracture. In posterior malleolus fractures involving less than $25 \%$ of the articular surface of the ankle, conservative treatment is typically effective without requiring internal fixation. ${ }^{[6,7]}$ The study found that after ankle fracture at the attachment point of the posterior tibiofibular ligament, posterior fixation of the ankle fracture can restore syndesmosis stability and reduce the occurrence of ankle joint complications. Some scholars believe that ${ }^{[8]}$ in posterior malleolus fractures involving more than $25 \%$ of the articular surface of the ankle, there is no clinical difference in conservative and surgical treatment. Another study ${ }^{[9]}$ reported that when the posterior malleolus fracture block makes up at least $25 \%$ of the ankle joint surface or is combined with subluxation of the talus and manual reduction failure, treatment should be carried out using open reduction surgery. However, in the clinic, the amount of articular surface area involved in the fracture is not the only indication for surgery. The type of fracture and degree of displacement as well as the lower tibiofibular joint stability are also considerations; and surgical operation is possible after ankle fracture displacement caused by it is also the clinical indications for surgery ${ }^{[3,10]}$. Fractures of the lower third of the tibia and of the posterior malleolus are different from a fracture of the posterior ankle. The present study found that $88 \%$

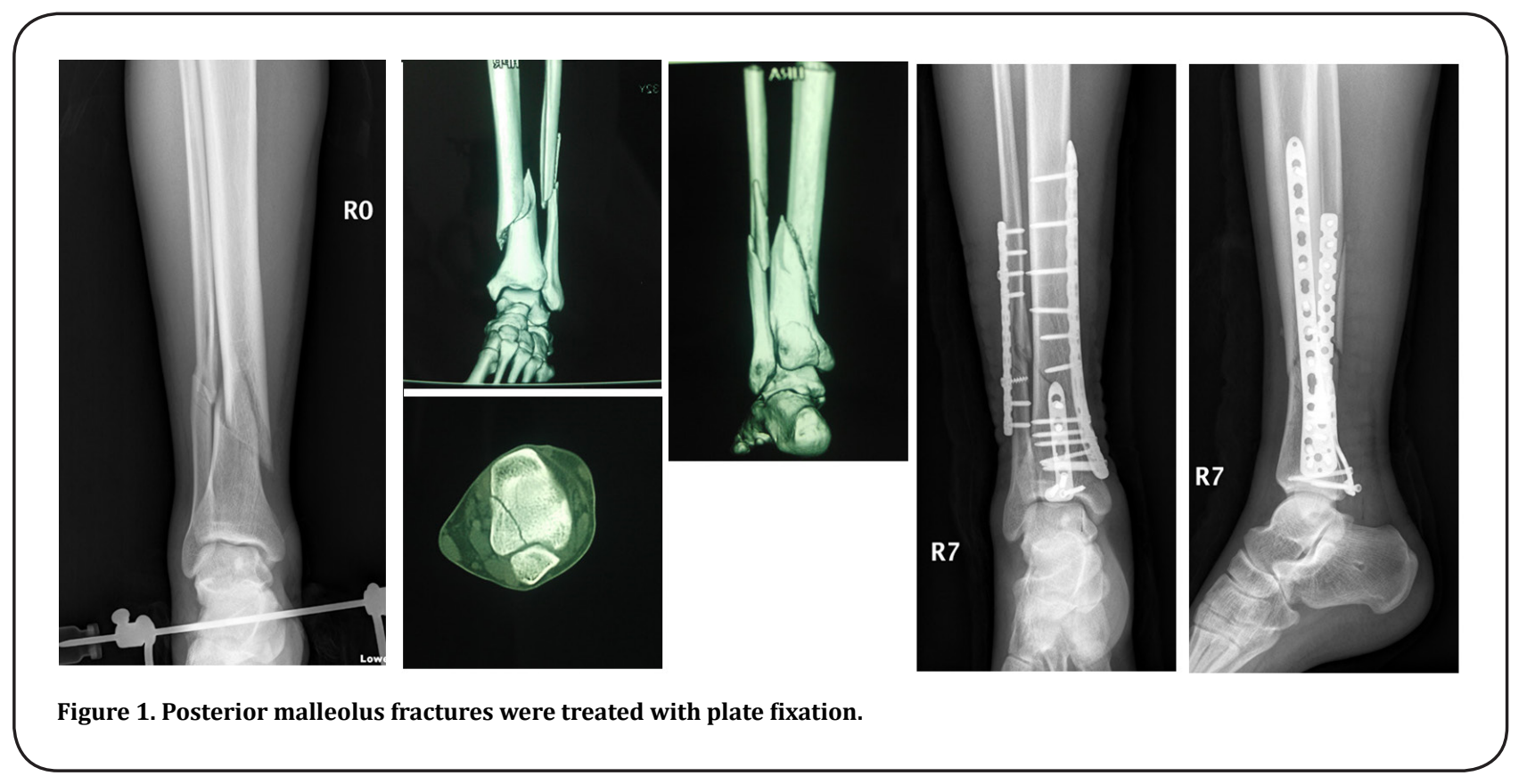



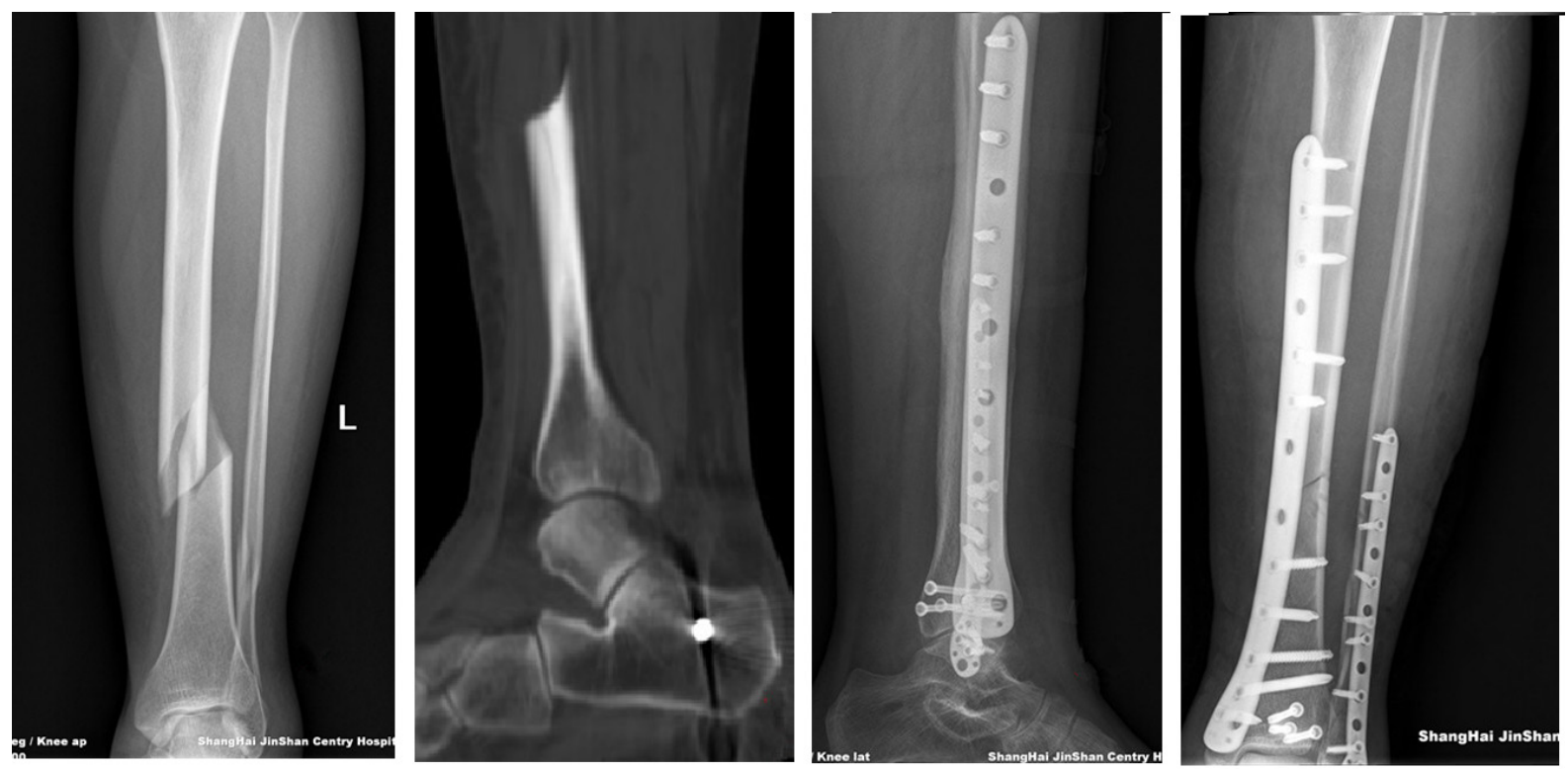

Figure 2. The posterior malleolus fractures were treated with three cannulated screws.

of patients had no obvious displacement. Therefore, clinical treatment of this condition should also have a special treatment plan.

A comparison of open reduction and internal fixation using three cannulated screws in the treatment of posterior malleolus fractures with an L-type plate

The purpose of this study was to reduce the incidence of postoperative ankle fracture displacement during functional exercise in patients with tibial fractures and also to compare the outcomes of the treatment of posterior ankle fractures in all patients whose fractured fragment of the posterior malleolus ranged from $25-50 \%$ of the articular surface.

Much research has been conducted on the clinical effects of posterior malleolus fractures. Reports on the condition of the ankle fracture block following plate fixation have been increasing in recent years. It has been reported ${ }^{[11]}$ that the strength of plate fixation in a posterior malleolus fracture block is greater than that of hollow screws. Therefore, patients who begin early postoperative passive and active functional ankle exercises do not have to worry about the fracture block shifting and affecting their recovery. The author anticipated that L-type plate fixation after ankle fracture block would have the following advantages: (1) for comminuted fractures, the block has a good fixation effect; (2) it improves the stability of the fixation; and (3) early functional exercise can promote rehabilitation and fracture healing. Clinical work has revealed that hollow screws have a stronger force on cortical and cancellous bone, but their holding force in a large ankle fragment is poor, especially in patients with osteoporosis. When posterior malleolar fractures are comminuted fractures, hollow screws often became loose, leading to easily induced late ankle joint separation. Therefore, comminuted posterior malleolus fractures that are large and occur in patients with osteoporosis should be repaired with plate fixation.

The treatment selection of either an L-type plate or three hollow screws in clinical practice

At present, there is no standard treatment the internal fixation of ankles fractured in two places. It has been suggested that the treatment of posterior ${ }^{[12]}$ fractures with cannulated screws can reduce surgical trauma, prevent damage to the blood supply of the posterior malleolus, and facilitate faster fracture healing and therefore an early return to functional exercise. At the same time, research shows ${ }^{[13]}$ that while screw fixation is more suitable for bone fractures, a large fracture block requires plate fixation after fracture of the ankle; the effect of plate fixation is superior to that of single screw fixation. Experimental ${ }^{[14]}$ studies have found good stability in the posterior facet of the ankle joint when a fracture of $25 \%$ of the articular surface was repaired with screw fixation, but it was also just as effective to use plate fixation at the $25 \%$ threshold. Dan Jin ${ }^{[15]}$ concluded that compared to screws, a plate is better at preventing an upward shift of the fracture block. Erdem et al. ${ }^{[16]}$ compared steel plate and screw fixation and reported that both a steel plate and screws can be firmly fixed to the fracture block, so the two groups were not statistically different. Connor et al. ${ }^{[17]}$ found 
that the plate fixation group had better results than the screw fixation group, but there was no difference between the two groups regarding ankle function; however, the incidence of postoperative traumatic arthritis in the screw fixation group was lower than that in the steel plate fixation group.

There are many confounding factors in these two groups. Our biomechanical study of the articular surface of posterior malleolar fractures that made up 15\% of the articular surface found that cannulated screw fixation and triangular L-type plate fixation were effective with no significant difference. When the size of the fragment was between $25-50 \%$ of the articular surface, cannulated screw fixation was biomechanically superior to L-type plate fixation even though triangle fixation can provide good stability. When determining the method of internal fixation of a posterior malleolus fracture, it is generally accepted that both screw and plate fixation have their own advantages and disadvantages, but the clinical effect of either is quite good. Screw fixation is minimally invasive and can achieve an effective fixation effect; however, from a biomechanical point of view, steel plate fixation has the advantage and is therefore more conducive to early functional exercise. In this group of cases, there was no significant difference in the clinical efficacy of the two types of internal fixation. Both were effective and feasible, reduced the incidence of surgical re-injury, had a high healing rate, and promoted a fast recovery. For stable fractures of the posterior malleolus, we suggest screw fixation to reduce tissue trauma. However, plate fixation is recommended for comminuted ankle fractures of the posterior malleolus with a size greater than a third of the bone, if the articular surface of the posterior malleolus fracture line is relatively long, or if the patient has osteoporosis to obtain more firm fixation so that patients may begin functional exercise as soon as possible postoperatively.

\section{ACKNOWLEDGMENT}

Fund program: This study is supported by the Shanghai science and Technology Commission (14411972600) and the Shanghai Municipal Health Bureau (2013-319).

\section{REFERENCES}

1. Baird, R. A., and Jackson, S. T. (1987) Fractures of the distal part of the fibula with associated disruption of the deltoid ligament. Treatment without repair of the deltoid ligament. The Journal of bone and joint surgery. American volume 69, 1346-1352

2. Wu, H. T., Hou, Z. Y., Zhang, Q., Zhao, H. T., Chen, W., Wu, W. J., Xue, P., Pan, J. S., and Zhang, Y. Z. (2008) [Clinical epidemiological analysis of adult spiral tibial shaft fracture associated with ipsilateral posterior malleolar fracture]. Zhonghua yi xue za zhi 88, 2166-2170

3. Purnell, G. J., Glass, E. R., Altman, D. T., Sciulli, R. L., Muffly, M. T., and Altman, G. T. (2011) Results of a computed tomography protocol evaluating distal third tibial shaft fractures to assess noncontiguous malleolar fractures. The Journal of trauma 71, 163-168

4. Stuermer, E. K., and Stuermer, K. M. (2008) Tibial shaft fracture and ankle joint injury. J Orthop Trauma 22, 107112

5. Kukkonen, J., Heikkila, J. T., Kyyronen, T., Mattila, K., and Gullichsen, E. (2006) Posterior malleolar fracture is often associated with spiral tibial diaphyseal fracture: a retrospective study. The Journal of trauma 60, 10581060

6. Mingo-Robinet, J., Lopez-Duran, L., Galeote, J. E., and Martinez-Cervell, C. (2011) Ankle fractures with posterior malleolar fragment: management and results. The Journal of foot and ankle surgery : official publication of the American College of Foot and Ankle Surgeons 50, 141-145

7. Gardner, M. J., Brodsky, A., Briggs, S. M., Nielson, J. H., and Lorich, D. G. (2006) Fixation of posterior malleolar fractures provides greater syndesmotic stability. Clinical orthopaedics and related research $447,165-171$

8. Huang, J. W., Luo, Y., Huang, Y. F., and Orthopaedics, D. O. (2013) Comparative study of clinical results of two treatment methods for lower $1 / 3$ tibial fractures associated with posterior malleolus fractures. Chinese Journal of Bone \& Joint Injury

9. Li, J., Liu, J., Chen, N., He, Q., and Li, Z. (2015) Diagnosis and treatment of the fracture of the middle and distal part of the tibia combined with posterior malleolus fracture. Journal of Practical Orthopaedics, 360-363

10. Berkes, M. B., Little, M. T., Lazaro, L. E., Pardee, N. C., Schottel, P. C., Helfet, D. L., and Lorich, D. G. (2013) Articular congruity is associated with short-term clinical outcomes of operatively treated SER IV ankle fractures. The Journal of bone and joint surgery. American volume 95, 1769-1775

11. Tang, Z. Y., Wang, W. L., and Tang, H. X. (2013) Surgical treatment of different size of posterior malleolar fracture. Chinese Journal of Bone \& Joint Injury

12. Song, J., Deng, X., Wang, X., Huang, D., and Chen, F. (2016) Surgical treatment on lower tibial fracture associated with posterior malleolus fracture. Journal of Clinical Orthopaedics 19, 192-192

13. Yin, J., Wang, X., Wang, C., Huang, J., Zhang, C., Chen, L., Wang, C., Geng, X., and Ma, X. (2016) Selection of internal fixation for posterior malleolar fractures. Chinese Journal of Orthopaedic Trauma 18, 545-549

14. Yao, L., Zhang, W., Yang, G., Zhu, Y., Zhai, Q., and Luo, C. (2014) Morphologic characteristics of the posterior malleolus fragment: a 3-D computer tomography based 
study. Archives of orthopaedic and trauma surgery 134, 389-394

15. Jin, D., Fu, S., Mei, G., Zhou, Z., Liu, S., Wang, S., and Liu, J. (2014) Comparative finite element analysis of fixations for posterior malleolar fracture. Chinese Journal of Orthopaedic Trauma 16, 1065-1069

16. Erdem, M. N., Erken, H. Y., Burc, H., Saka, G., Korkmaz,
M. F., and Aydogan, M. (2014) Comparison of lag screw versus buttress plate fixation of posterior malleolar fractures. Foot \& ankle international 35, 1022-1030

17. O'Connor, T. J., Mueller, B., Ly, T. V., Jacobson, A. R., Nelson, E. R., and Cole, P. A. (2015) "A to p" screw versus posterolateral plate for posterior malleolus fixation in trimalleolar ankle fractures. J Orthop Trauma 29, e151-156 